\title{
Incisional Hernia in Renal Transplant Recipients: A Systematic Review
}

Simson N, Parker S.G, Stonier T, Halligan S, Windsor A.C.J.

Corresponding Author:

Nick Simson

60 Stile Hall Gardens

London, United Kingdom

W4 3BU

+447962248838

njsimson@gmail.com

\section{$\underline{\text { Abstract }}$}

\section{Background}

Incisional hernia follows midline laparotomy in 8 to $20 \%$ of cases but the rate following lateral incision is not well documented. This systematic review summarises incisional hernia rate after open renal transplant.

\section{Method}

We searched EMBASE, MEDLINE, and the Cochrane Library databases from January 2000 to November 2016 inclusive. The outcomes included in our analysis were the post-transplant incisional hernia rate, significant patient risk factors for incisional hernia, the definition of incisional hernia used, the method used to detect incisional hernia and the incision used for transplantation.

\section{Results}

Eight retrospective case series were identified, three describing renal transplant recipients and five describing incisional hernia repairs post renal transplant. All reported the incisional hernia rate post-renal transplant at the host institution. The hernia rate ranged from 1.1 to $7.0 \%$, with a mean of $3.2 \%$. Factors associated with incisional hernia were $\mathrm{BMI}>30$, Age $>50$, cadaveric graft, and reoperation through the same incision. 


\section{Conclusion}

Despite the significant co-morbidity of renal transplant recipients, the incisional hernia rate post-renal transplant is significantly lower than post midline laparotomy. The reasons for this are discussed. This demonstrates the importance of operative technique, local tissue quality and biomechanical factors in the formation of incisional hernia.

\section{Background}

Incisional hernia remains one of the most common postoperative complications following abdominal surgery. Current data suggests an incidence between 8 to 20\% (1),(2),(3),(4),(5). Renal transplant recipients have a theoretically increased risk of developing wound complications postoperatively, given their immunosuppressive regime, prolonged periods of dialysis and often complex comorbidity (6),(7). However, the rates of incisional hernia in these patients are believed to be far lower than for midline abdominal operations. Single-centre studies have estimated the incidence of incisional hernia following renal transplant surgery to be between $3.2 \%$ (8) and 7\% (9) at 4.5 years follow up. However, at the time of writing there is no published systematic review that reports the incisional hernia rate through a post renal transplant incision across several institutions. As academic hernia surgeons research abdominal wall dynamics and incisional hernia rates, the flank incisional hernia rate after renal transplant should be known with precision. Accordingly, via systematic review of the available primary literature we aimed to determine the incisional hernia rate following renal transplant and to assess the risk factors for hernia development in these patients. 


\section{Methods}

\section{Reporting}

This systematic review was performed and reported in line with the Preferred Reporting Items for Systematic Reviews and Meta-Analysis (PRISMA) statement (10). Ethical permission is not required by our centre for systematic reviews of available primary literature.

\section{Inclusion criteria for studies}

We aimed to identify case series and observational studies reporting the incisional hernia rate following open renal transplant from January 2000 to November 2016 inclusive. Only studies reporting human patients and written in the English language were included.

\section{Inclusion and exclusion criteria for participants}

The target condition was incisional hernia following open renal transplant. Studies relating to nephrectomy, donor nephrectomy, non-renal transplantation, non-incisional hernia were excluded. Studies including augmented mesh closure post-transplant were excluded. So as to eliminate any learning curve or centres with limited experience, we excluded any study reporting less than 200 individual patients.

\section{Search strategy and string}


A surgical research trainee, NS, used the ProQuest search engine (ProQuest DialogTM, Ann Arbor, Michigan, USA) to search the EMBASE, MEDLINE, and the Cochrane Library databases from $1^{\text {st }}$ January 2000 to $1^{\text {st }}$ November 2016 inclusive. The search filters "human studies" and "written in English" were applied. Our search string combined three search terms using title and abstract keywords as well as subject (thesaurus) terms to identify articles for inclusion as follows:

- All titles, abstracts and subject terms containing the keywords "renal" OR "kidney".

- All titles, abstracts and subject terms containing the keyword "transplant".

- All titles, abstracts and subject terms containing the keywords "hernia" OR "incisional".

Our complete search string is shown in Appendix 1

\section{Citation management and Screening}

After applying the search filters, ProQuest issued the search results in PDF format, which were then stored by NS. Two researchers, NS and TS, then independently screened the titles and abstracts of the citation results and categorised each as 'clearly unsuitable', 'uncertain' or 'definitely possible'. Duplicate citations were removed. The independent screening results were then compared and any discrepancies resolved by face-to-face discussion. The full text of all the remaining 'uncertain' and definitely possible' citations were then retrieved and hand searched by NS and TS, and any paper 
deemed ultimately unsuitable discarded. A senior member of the research team, SGP, was available for advice throughout the search. Ultimately, search results were discussed face-to-face and studies to be included in the review finalised.

\section{Data extraction}

NS and TS both extracted data from all included studies. Extracted data were recorded into a data extraction sheet (Microsoft Excel for Mac 2011 Version 14.4.7, Microsoft Corporation, Washington, USA) designed specifically for the systematic review. Extracted data were categorised according to: study design; primary outcome and secondary outcomes. The Methodological Index of Nonrandomized Studies (MINORS) (11) was used to evaluate methodologic quality and potential bias of selected articles. Both NS and TS scored the articles independently.

For study design, we recorded; the study type, the number of patients included, the patient demographics (age, gender, body mass index), the method of data collection (prospective versus retrospective), country of publication, year of publication, length of follow-up, statistically significant risk factors for hernia development, and the methodological quality of the study as assessed by MINORS (11) criteria.

\section{Clinical outcome}

For our primary outcome, we recorded the rate of incisional hernia formation and the time to hernia formation if reported. We also recorded definitions for incisional hernia 
occurrence if reported and the method used for hernia detection (eg. Clinical examination, USS or CT scanning). Our secondary outcomes were any risk factors for incisional hernia reported as statistically significant by the authors. We recorded the pvalues for these and the statistical methods used to show significance. Where possible, we also included postoperative complication rates following transplantation. Postoperative complications were grouped into intra-operative, early post-operative, and late post-operative. Early complications were defined as those occurring within 30 days of surgery and late complications as those thereafter.

\section{Data synthesis, analysis and reporting}

For data that were obviously heterogeneous by visual inspection, we used basic statistical methods and narrative to describe our findings. Tables were used to display results according to our data collection categories. We anticipated a significant amount of heterogeneity in terms of the variables recorded and the follow up period. However, we reported all available data to avoid selection and reporting bias. If possible we intended to combine studies and perform univariate and multivariate analysis to identify predictor variables for incisional hernia following renal transplantation.

\section{$\underline{\text { Results }}$}

\section{Study Selection}

A total of 961 potential articles were identified from the initial literature search. 754 articles remained after the language, date and human trial filters were applied. Using 
the inclusion criteria described above, 724 were eliminated after title and abstract review. The full text of 30 articles was obtained of which 23 were rejected. The references of the full text articles were interrogated and one additional suitable study was included. Thus eight articles were included for final analysis (Figure 1).

All eight studies were retrospective case series. Three described unselected renal transplant patients and five described incisional hernia repair following renal transplantation. These five papers were included as they reported the incisional hernia rates for the entire unselected population of renal transplants recipients at the host institution.

\section{Study design and Study Quality}

A total of 12,035 renal transplant recipients were reported by the eight studies. The number of patients included in each individual study ranged from 371 to 2499 with a mean of 1504. Three studies were carried out in Brazil (12),(13),(7) and two were carried out in the USA (14),(15). In seven studies the data were collected retrospectively from patient medical record review. In the remaining study, data were entered into a prospectively collected database but analysed retrospectively(9). Study quality, as assessed by the MINORS criteria, ranged from 6 to 10 (out of a total of 16) with a mean score of 7.3. Five of the studies only had a MINORS score of $6(6),(12),(13),(15),(16)$. The two most recent studies had the highest scores of 10 (8),(9). Based on MINORS criteria, six studies were limited by their inadequate description of follow up(6),(12),(13),(14),(15),(16), five by their inability to account for loss to follow up(6),(12),(13),(15),(16), the patients from all eight studies were 
assessed by the primary surgical team in the outpatient clinic, subjecting the outcomes to reporting bias.

Where specified, the mean or median age of patients ranged from 31 to 54 years, with a mean age of 45 years for the studies overall. In the seven studies where gender was specified, there was a higher proportion of males, with a mean value of 62 percent of patients being male. Five of the seven studies that specified donor type, had a higher preponderance of cadaveric donors, with a range of 51 to 89 percent of donors cadaveric, whereas two studies reported predominantly live donors, with 65 (6) and 98 percent live donors (8). The mean percentage of cadaveric donors was 51 percent. BMI was specified in only two studies, and was 25.7 (8) and 27.8 (9) respectively.

Figure 1. Selection of articles for review as per PRISMA guidelines

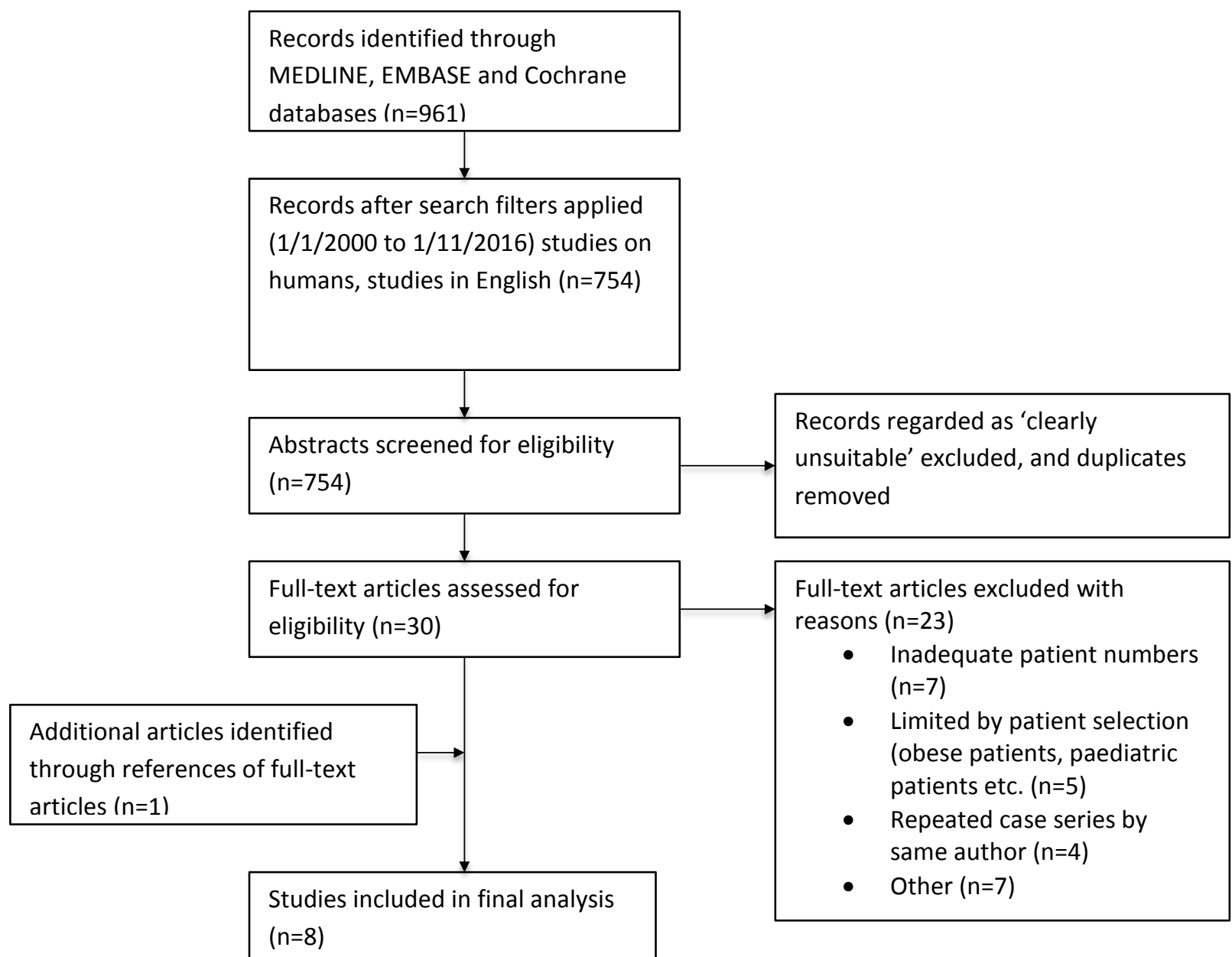




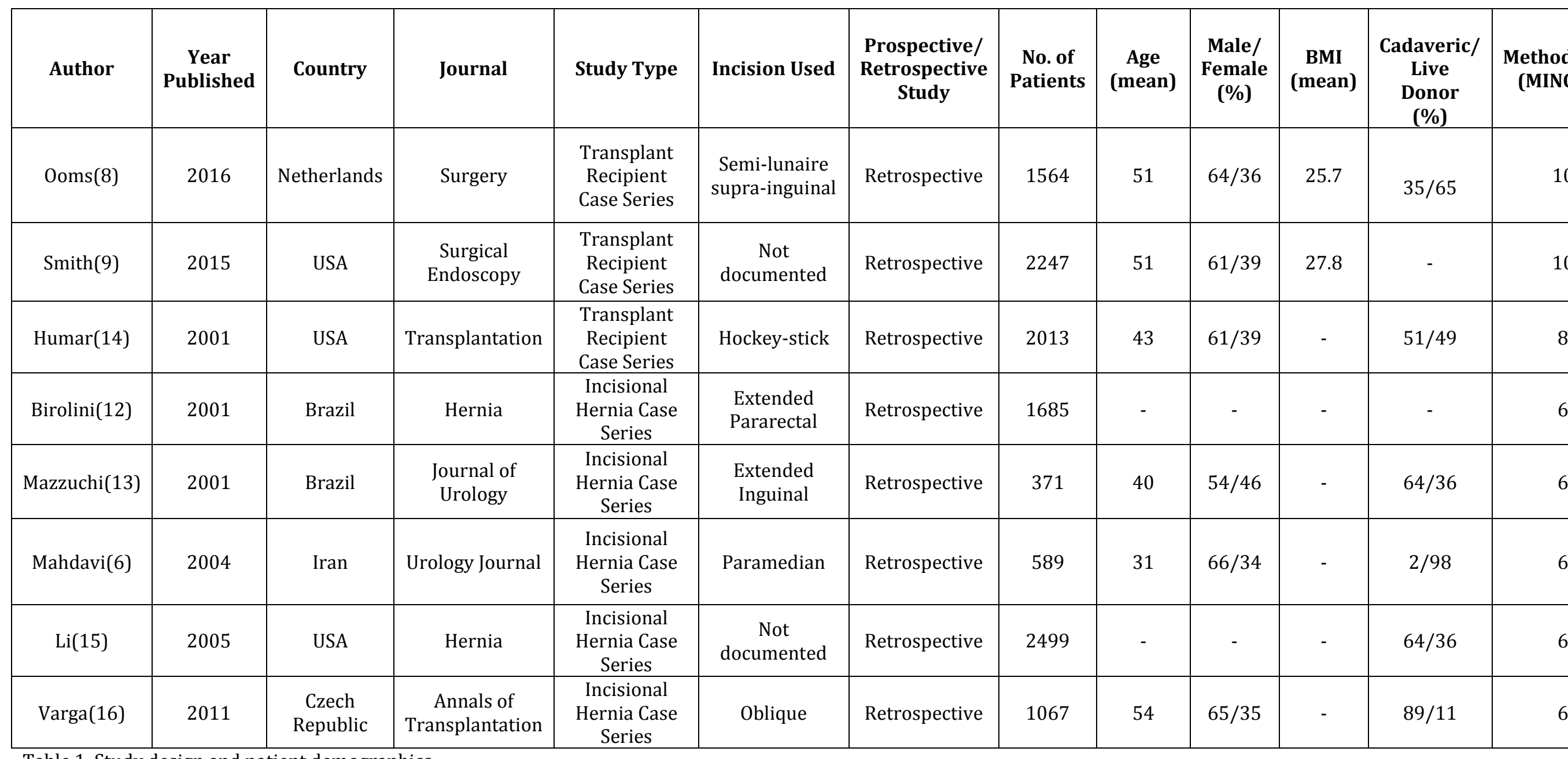

Table 1. Study design and patient demographics. 


\begin{tabular}{|l|c|c|c|c|c|l|}
\hline \multicolumn{1}{|c|}{ Author } & $\begin{array}{c}\text { Number } \\
\text { of } \\
\text { patients } \\
\text { with } \\
\text { incisional } \\
\text { hernia }\end{array}$ & $\begin{array}{c}\text { Incisional } \\
\text { hernia } \\
\text { rate (\%) }\end{array}$ & $\begin{array}{c}\text { Time for } \\
\text { hernia } \\
\text { development } \\
\text { (weeks) }\end{array}$ & $\begin{array}{c}\text { Definition of } \\
\text { incisional } \\
\text { hernia } \\
\text { described? } \\
\text { (Yes/No) }\end{array}$ & $\begin{array}{c}\text { Length of } \\
\text { follow up } \\
\text { (months) }\end{array}$ & $\begin{array}{c}\text { Method used to detect } \\
\text { incisional hernia }\end{array}$ \\
\hline Ooms(8) & 50 & 3.2 & 68 & No & 59 & $\begin{array}{l}\text { Clinical Examination } \\
\text { +/- imaging if in doubt }\end{array}$ \\
\hline Smith(9) & 157 & 7.0 & - & No & 55 & Clinical Examination \\
\hline Humar(14) & 73 & 3.6 & 55.6 & No & 60 & Not stated \\
\hline Birolini(12) & 19 & 1.1 & - & No & - & Not stated \\
\hline Mazzucchi(13) & 14 & 3.8 & 8.6 & No & - & Clinical Examination \\
\hline Mahdavi(6) & 16 & 3.0 & 6.8 & No & - & Not stated \\
\hline Li(15) & 41 & 1.6 & - & No & - & Not stated \\
\hline Varga(16) & 20 & 2.6 & 76 & No & - & Not stated \\
\hline
\end{tabular}

Table 2. Incisional hernia formation rate post renal transplant

\begin{tabular}{|c|c|c|c|}
\hline Author & $\begin{array}{c}\text { Reported risk factors for incisional } \\
\text { hernia }\end{array}$ & $\begin{array}{l}\text { Univariate } \\
\text { Multivariate } \\
\text { Analysis }\end{array}$ & P value \\
\hline Ooms(8) & $\begin{array}{l}\text { Female } \\
\text { BMI }>30 \\
\text { Smoking History } \\
\text { Concurrent abdominal wall hernia } \\
\text { Left-sided implantation } \\
\text { Duration of surgery } \\
\text { Multiple explorations of iliac fossa } \\
\text { Female } \\
\text { Smoking History } \\
\text { BMI }>30 \text {, } \\
\text { Multiple exploration of iliac fossa, } \\
\text { Duration of operation, } \\
\text { Concurrent abdominal wall hernia }\end{array}$ & $\begin{array}{l}\begin{array}{l}\text { Univariate } \\
\text { analysis }\end{array} \\
\text { Multivariate } \\
\text { analysis }\end{array}$ & $\begin{array}{l}0.006 \\
<0.001 \\
0.032 \\
0.007 \\
0.046 \\
0.02 \\
0.001 \\
\\
0.002 \\
0.019 \\
<0.001 \\
0.026 \\
0.014 \\
0.009\end{array}$ \\
\hline $\operatorname{Smith}(9)$ & $\begin{array}{l}\text { BMI>25, } \\
\text { Surgical site infection } \\
\text { Delayed graft function, } \\
\text { Absence of MMF inhibitor } \\
\text { Absence of calcineurin inhibitor } \\
\text { Surgical Site Infection, } \\
\text { Absence of MMF, }\end{array}$ & $\begin{array}{l}\text { Univariate } \\
\text { analysis } \\
\text { Multivariate } \\
\text { analysis }\end{array}$ & $\begin{array}{l}0.02 \\
<0.001 \\
0.003 \\
0.001 \\
0.002 \\
\\
<0.001 \\
<0.001\end{array}$ \\
\hline Humar(14) & $\begin{array}{l}\text { BMI }>30 \\
\text { Age }>50 \text { years, } \\
\text { Re-operation through same incision, } \\
\text { MMF vs Azathioprine }\end{array}$ & $\begin{array}{l}\text { Multivariate } \\
\text { analysis }\end{array}$ & $\begin{array}{l}0.0003 \\
0.01 \\
0.0001 \\
0.0004 \\
\end{array}$ \\
\hline
\end{tabular}

Table 3. Significant risk factors for development of incisional hernia in renal transplant recipients 


\section{Incisional Hernia}

The mean rate of incisional hernia across all studies was 3.2\% (390/12035), ranging from $1.1 \%(19 / 1685)(12)$ to $7 \%(157 / 2247)$ (9) for the individual studies. Length of follow up was only reported in three out of eight studies (8),(9),(14). Two studies reported 10-year incisional hernia rates, which were 7\% (9) and 4.4\% (8). The average (mean or median) time taken for hernia to develop was recorded in five studies and ranged from 6.8 weeks to 76 weeks (Table 2), with a mean of 43 weeks. Six studies described the incision used for renal transplantation; three studies used an oblique incision described separately as either an oblique incision(16), an extended inguinal(13), or a semi-lunaire supra-inguinal incision(8). The remaining three studies used a non-midline longitudinal incision described as pararectal(12), paramedian(6) and hockey stick(14). None of the studies defined incisional hernia. Five studies did no mention how incisional hernia was detected. Two studies(9),(13) used clinical examination to detect incisional hernia and one study(8) adopted US or CT imaging where the clinical diagnosis of incisional hernia was in doubt.

\section{Risk Factors for Incisional Hernia}

Three studies (8),(9),(14) analysed risk factors for hernia development using multivariate analysis. The reported significant risk factors for hernia development were Body Mass Index (BMI) >30 (p<0.001) (8),(14) surgical site infection (SSI) $(p<0.001)$, absence of mycophenalate mofetil $(p<0.001)(9)$, Age $>50$ years $(p=0.01)(14)$, reoperation through the same incision $(p=0.0001)(8),(14)$ mycophenalate mofetil compared to azathioprine immunosuppression $(p=0.0004)(14)$, female sex 
$(p=0.002)(8)$, concurrent abdominal wall hernia $(p=0.009)(8)$, history of smoking $(p=0.019)(8)$, and prolonged duration of surgery $(p=0.014)(8),($ Table 3$)$. A weighted statistical analysis of the risk factors from all the studies for incisional hernia development was not possible because of the lack of the unacceptable heterogeneity of the reported data.

\section{Post-operative complications}

Post renal transplantation complication rates were reported poorly and could not be categorised as we had intended. Ooms et al.(8) reported a re-operation rate of 19\% (301 of 1564 patients) having re-intervention for bleeding, urological complications, or transplanted kidney removal. Humar et al.(14) reported a post-operative wound infection rate of $4.8 \%$ (97 of 2013 transplant recipients), with a superficial wound infection rate of 3.8\% (76 of 2013 transplant recipients) and a deep wound infection rate of $1.0 \%$ (21 of 2013 transplant recipients). Post transplant complications were not reported by any other series.

\section{$\underline{\text { Discussion }}$}

Incisional hernia following abdominal surgery is reported at between 8 to $20 \%$. A recent systematic review reported the overall midline incisional hernia rate at $12.8 \%$, with an overall increase from $8 \%$ to $16 \%$ over the last thirty years (17). In this systematic review, we focus specifically on lateral hernias following renal transplantation, which has been relatively under-investigated. We found a mean 
incisional hernia rate after renal transplant of 3.2\%, a figure very significantly lower than that for midline laparotomy.

Much of the literature on incisional hernia has focused on comorbidities that may predispose a patient to herniogenesis. In our review, the included studies that performed univariable and multivariable analysis showed repeatedly that BMI $>30$, age $>50$, cadaveric graft and reoperation through the same incision predispose to incisional hernia. The use of mycophenalate mofetil (MMF) for post-transplant immunosuppression remains a contentious issue as Humar et al.(14) list MMF as a risk factor when compared to azathioprine, whereas Smith et al.(9) link absence of MMF to development of incisional hernia. In the literature, smoking(18), diabetes(19), previous wound infection(20), steroid use(21), and increased BMI(22) have all been shown to increase the probability of incisional hernia after intra-abdominal surgery. Consequently, renal transplant patients would be expected to be at higher risk of incisional hernia due to prolonged periods of dialysis prior to transplantation, immunosuppression post transplantation, and a higher prevalence of co-morbidities such as obesity, diabetes, chronic obstructive pulmonary disease, and hypertension(7),(23). However, despite increased comorbidity we found the opposite is true; incisional hernia is significantly less frequent than for midline laparotomy. It seems likely, therefore, that incisional hernia must have a higher dependency on factors specific to the abdominal incision rather than general patient comorbidity. These dependent factors could include; the location of the abdominal incision, the technique used to close the incision, the quality and strength of the abdominal wall tissue around the incision site, and the local biomechanical forces around the closed incision.

The studies included in our review used a variety of incisions for renal transplantation including para-medial incision, oblique incision, extended inguinal incision, para-rectal 
incision, and hockey-stick incision. Previous systematic reviews of general surgical patients (non-transplant patients) have shown that paramedian and transverse abdominal incisions have statistically lower incisional hernia rates(24) when compared to midline incisions. Paramedian incisions are similar to hockey-stick and pararectal incisions as all use the semilunar ligament, at the lateral border of the rectus abdominis, to gain access to the abdominal cavity. The oblique and extended inguinal incisions are also similar and extend laterally parallel to the muscle fibres of the external oblique. A study comparing the incisional hernia rates for hockey stick versus oblique incisions has shown the oblique incision to have statistically lower herniation rates(25). Indeed, the evidence for transverse laparotomies demonstrating low complication rates at the wound site is well-known and the European guidelines recommend transverse incisions where possible as not only is the incisional hernia rate decreased but so is postoperative analgesia (26),(27).

Not all of our included studies reported their incision closure technique. The studies that did used a continuous non-absorbable suture to close the abdominal fascia. There is no mention of using the 'small bites' technique or closure with mesh augmentation. This is understandable as even though there is strong evidence showing the 'small bites' closure technique reduces incisional hernia rates in midline laparotomies there is no evidence that this is true for oblique or paramedical incisions. As for closure with mesh augmentation, even though European guidelines do state that mesh is advised for 'highrisk' closures(26), most transplant surgeons do not use mesh for primary closure due to the risk of infection in immunosuppressed patients. Transplant surgeons should be meticulous about their primary closure technique as flank hernias can be difficult to close, with some hernia specialists using bone anchors to the iliac crest to establish a strong repair(28),(29). 
The quality and strength of the abdominal wall structures at the incision site must influence incisional hernia rates. Comorbidities will affect the health, quality and strength of abdominal wall structures (muscles and ligaments). However, we found that comorbidities are likely to play only a small part in herniogenesis, as transplant patients, assumed to have multiple comorbidies, exhibited a relatively low incisional hernia rate. Apart from anatomical incision site and method of wound closure, future research should also focus on the direction and strength of the abdominal wall biomechanical forces as well as tissue structure and orientation at the incision site. Research in these areas is still in its infancy. We know that a lower collagen I/III ratio reduces tissue tensile strength and predisposes to herniogensis(30). Biomechanical studies of the linea alba and the rectus sheath show reduced tensile strength of scar tissue and anisotropy with increased strength in the transverse direction compared to longitudinal (31),(32). Abdominal wall compliance and strain have been found to be greater in the longitudinal direction(33). How these discoveries affect herniogenesis and surgical repair remains unclear and further research comprising histological and biomechanical analysis of abdominal wall structures is warranted.

Importantly our review also highlights a lack of a standardised definition for incisional hernia and no standardised method of incisional hernia diagnosis (only one study used imaging to aid diagnosis(8)). Both of these phenomena predispose to unstandardised data and variability in hernia diagnosis. In addition, most studies did not specify the length of follow up. Accordingly, it seems that a universally accepted definition of incisional hernia; a standardised test method to detect such hernia; and standardised assessment time-frame for diagnosis is required.

\section{Conclusion}


The reported incidence of incisional hernia following renal transplantation averages $3.2 \%$, ranging from 1.1 to $7 \%$, a rate significantly lower than that following midline laparotomy. As end-stage renal failure patients have multiple comorbidities(7),(23) we conclude that incisional hernia cannot be principally dependent on comorbidity but must be dependent on the local factors that influence biomechanics and wound healing at the incisional site. Lastly, our review found that a standardised definition of incisional hernia, diagnostic methods, and duration of follow-up is required in hernia research to reduce the variability of reported data.

\section{References}

1. Burger J, Luijendijk R, Hop WCJ, et al. Long-term Follow-up of a Randomized Controlled Trial of Suture Versus Mesh Repair of Incisional Hernia. Ann Surg $2004 ; 240(4): 578-85$.

2. Diener MK, Voss S, Jensen K, et al. Elective Midline Laparotomy Closure: the INLINE systematic review and meta-analysis. Ann Surg 2010;251(5):843-56.

3. Riet M, Steyerberg EW, Nellensteyn J, et al. Meta-analysis of techniques for closure of midline abdominal incisions. Br J Surg 2002;89(11):1350-6.

4. Van Ramshorst GH, Eker HH, Hop WCJ, et al. Impact of incisional hernia on healthrelated quality of life and body image: A prospective cohort study. Am J Surg 2012;204(2):144-50.

5. Fink C, Baumann $\mathrm{P}$, Wente $\mathrm{MN}$, et al. Incisional hernia rate 3 years after midline laparotomy. Br J Surg 2014;101:51-4.

6. Mahdavi R, Mehrabi M. Incisional hernia after renal transplantation and its repair with propylene mesh. Urol J. 2004;1:259-62. 
7. Antonopoulos IM, Nahas WC, Mazzucchi E, et al. Is polypropylene mesh safe and effective for repairing infected incisional hernia in renal transplant recipients? Urology 2005;66(4):874-7.

8. Ooms LS, Verhelst J, Jeekel J, et al. Incidence, risk factors, and treatment of incisional hernia after kidney transplantation: An analysis of 1,564 consecutive patients. Surgery 2016;159(5):1407-11.

9. Smith CT, Katz MG, Foley D, et al. Incidence and risk factors of incisional hernia formation following abdominal organ transplantation. Surg Endosc 2015;29:398404.

10. Liberati A, Altman DG, Tetzlaff J, et al. The PRISMA statement for reporting systematic reviews and meta-analyses of studies that evaluate health care interventions: explanation and elaboration. J Clin Epidemiol 2009;62(10):e1-34.

11. Slim K, Nini E, Forestier D, et al. Methodological index for non-randomized studies (Minors): Development and validation of a new instrument. ANZ J Surg 2003;73(9):712-6.

12. Birolini C, Mazzucchi E, Utiyama EM, et al. Prosthetic repair of incisional hernia in kidney transplant patients. A technique with onlay polypropylene mesh. Hernia 2001;5:31-5.

13. Mazzuchi E, Carlos Nahas W, Antonopoulos I, et al. Incisional hernia and its repair with polypropylene mesh in renal transplant recipients. J Urol. 2001;166:816-9.

14. Humar A, Ramcharan T, Denny R, et al. Are wound complications after a kidney transplant more common with modern immunosuppression? Transplantation 2001;72(12):1920-3.

15. Li EN, Silverman RP, Goldberg NH. Incisional hernia repair in renal transplantation patients. Hernia 2005;9(3):231-7. 
16. Varga M, Matia I, Kucera M, et al. Polypropylene mesh repair of incisional hernia after kidney transplantation: single-center experience and review of the literature. Ann Transpl 2011;16(3):121-5.

17. Bosanquet DC, Ansell J, Abdelrahman T, et al. Systematic review and metaregression of factors affecting midline Incisional hernia rates: Analysis of 14618 Patients. PLoS One 2015;10(9):1-18.

18. Sorensen LT, Hemmingsen UB, Kirkeby LT. Smoking is a risk factor for incisional hernia. Arch Surg 2015;140:119-23.

19. Hornby ST, McDermott FD, Coleman M, et al. Female gender and diabetes mellitus increase the risk of recurrence after laparoscopic incisional hernia repair. Ann $\mathrm{R}$ Coll Surg Engl. 2015;97(2):115-9.

20. Cobb WS, Warren JA, Ewing JA, et al. Open retromuscular mesh repair of complex incisional hernia: Predictors of wound events and recurrence. J Am Coll Surg 2015;220(4):606-13.

21. Sugerman HJ, Kellum JM, Reiness HD, et al. Greater Risk of Incisional Hernia with Morbidly Obese than Steroid-Dependent Patients and Low Recurrence With Prefascial Polypropylene Mesh. Am J Surg 1996;171:80-4.

22. Desai KA, Razavi SA, Hart AM, et al. The Effect of BMI on Outcomes Following Complex Abdominal Wall Reconstructions. Ann Plast Surg 2015;76(Suppl 4):p S295-S297.

23. Wu C, Evans I, Joseph R, Shapiro R, Tan H, Basu A, et al. Comorbid conditions in kidney transplantation: association with graft and patient survival. J Am Soc Nephrol 2005;16(11):3437-44.

24. Bickenbach KA, Karanicolas PJ, Ammori JB, et al. Up and down or side to side? A systematic review and meta-analysis examining the impact of incision on 
outcomes after abdominal surgery. Am J Surg 2013;206(3):400-9.

25. Nanni G, Tondolo V, Citterio F, et al. Comparison of oblique versus hockey-stick surgical incision for kidney transplantation. Transplant Proc 2005;37(6):247981.

26. Muysoms FE, Antoniou SA, Bury K, et al. European Hernia Society guidelines on the closure of abdominal wall incisions. Hernia 2015;19(1):1-24.

27. Brown S, Goodfellow P. Transverse versus midline incisions for abdominal surgery. Cochrane Database Syst Rev 2005;(4).

28. Blair LJ, Cox TC, Huntington CR, et al. Bone anchor fixation in abdominal wall reconstruction: A useful adjunct in suprapubic and para-iliac hernia repair. Am Surg 2015;81(7):693-7.

29. Patten LC, Awad SS, Berger DH, Fagan SP. A novel technique for the repair of lumbar hernias after iliac crest bone harvest. Am J Surg 2004;188(1):85-8.

30. Junge K, Klinge U, Rosch R, et al. Decreased collagen type I/III ratio in patients with recurring hernia after implantation of alloplastic prostheses. Langenbecks Arch Surg 2004;389(1):17-22.

31. Hollinsky C, Sandberg S. Measurement of the tensile strength of the ventral abdominal wall in comparison with scar tissue. Clin Biomech 2007;22:88-92.

32. Anurov M V, Titkova SM, Oettinger AP. Biomechanical compatibility of surgical mesh and fascia being reinforced: Dependence of experimental hernia defect repair results on anisotropic surgical mesh positioning. Hernia 2012;16:199-210.

33. Śmietański M, Bury K, Tomaszewska A, et al. Biomechanics of the front abdominal wall as a potential factor leading to recurrence with laparoscopic ventral hernia repair. Surg Endosc Other Interv Tech 2012;26(5):1461-7. 
Appendix 1 - Search Strategy to identify initial papers

\section{Search Strategy}

Databases: Embase $\circledast$, MEDLINE®

\begin{tabular}{|c|c|c|}
\hline Set\# & Searched for & Results \\
\hline S1 & ti,ab,su(renal* OR kidney*) & $2332570^{\star}$ \\
\hline S2 & ti,ab,su(transplant*) & $2690710^{\star}$ \\
\hline s3 & ti,ab,su(hernia* OR incisional*) & $204353^{\star}$ \\
\hline S4 & s1 AND s2 AND s3 & $961^{\circ}$ \\
\hline S5 & $\begin{array}{l}\text { (s1 AND s2 AND s3) and pd(20000101-20161101) and } \\
\text { human(yes) and la.exact("English") }\end{array}$ & $754^{\circ}$ \\
\hline
\end{tabular}

* Duplicates are removed from the search, but included in the result count.

-Duplicates are removed from the search and from the result count. 